\title{
Profile-Guided Transaction Coalescing-Lowering Transactional Overheads by Merging Transactions
}

\author{
SRDAN STIPIĆ, VESNA SMILJKOVIĆ, OSMAN UNSAL, ADRIÁN CRISTAL, \\ and MATEO VALERO, Barcelona Supercomputing Center, Spain
}

\begin{abstract}
Previous studies in software transactional memory mostly focused on reducing the overhead of transactional read and write operations. In this article, we introduce transaction coalescing, a profile-guided compiler optimization technique that attempts to reduce the overheads of starting and committing a transaction by merging two or more small transactions into one large transaction. We develop a profiling tool and a transaction coalescing heuristic to identify candidate transactions suitable for coalescing. We implement a compiler extension to automatically merge the candidate transactions at the compile time. We evaluate the effectiveness of our technique using the hash table micro-benchmark and the STAMP benchmark suite. Transaction coalescing improves the performance of the hash table significantly and the performance of Vacation and SSCA2 benchmarks by $19.4 \%$ and 36.4\%, respectively, when running with 12 threads.
\end{abstract}

Categories and Subject Descriptors: D.3.4 [Programming Languages]: Processors-Compilers

General Terms: Algorithms, Performance

Additional Key Words and Phrases: Coalescing, optimization, transactional memory

ACM Reference Format:

Stipić, S., Smiljković, V., Unsal, O., Cristal, A., and Valero, M. 2013. Profile-guided transaction coalescinglowering transactional overheads by merging transactions. ACM Trans. Architec. Code Optim. 10, 4, Article 50 (December 2013), 18 pages.

DOI: http://dx.doi.org/10.1145/2555289.2555306

\section{INTRODUCTION}

Transactional Memory (TM) [Herlihy and Moss 1993; Harris et al. 2010] is a concurrency mechanism that simplifies the development of multithreaded applications. TM is much easier to use than locks because it does not suffer from most of the problems associated with locks (deadlocks, multiple releases of a lock, noncomposability, etc.). Most of the current TM systems are implemented in software as a library or as a compiler extension. In order to support concurrent execution of transactions, a Software TM (STM) system manages transactional metadata during transactional execution. This creates overheads related to transaction initialization, versioning, read/write instrumentation, and so on. At the beginning of each transaction, the STM system initializes transactional metadata (read and write sets) and creates a checkpoint of the current thread state (the register file and local variables). If the transaction aborts, due to a conflict with another transaction, the checkpoint is used to restore the state from the beginning of the transaction. Otherwise, it commits changed shared data and associated metadata. If transactions in an application are numerous and small, STM

Authors' addresses: S. Stipic, Barcelona Supercomputing Center - Transactional MemoryGran Capita 2 Nexus I Oficina 304 Barcelona Barcelona, Spain; email: srdjan.stipic@bsc.es; srdjan.stipic@gmail.com. Permission to make digital or hard copies of part or all of this work for personal or classroom use is granted without fee provided that copies are not made or distributed for profit or commercial advantage and that copies show this notice on the first page or initial screen of a display along with the full citation. Copyrights for components of this work owned by others than ACM must be honored. Abstracting with credit is permitted. To copy otherwise, to republish, to post on servers, to redistribute to lists, or to use any component of this work in other works requires prior specific permission and/or a fee. Permissions may be requested from Publications Dept., ACM, Inc., 2 Penn Plaza, Suite 701, New York, NY 10121-0701 USA, fax +1 (212) 869-0481 or permissions@acm.org.

(c) 2013 ACM 1544-3566/2013/12-ART50 $\$ 15.00$

DOI: http://dx.doi.org/10.1145/2555289.2555306 
overheads accumulate rapidly. The overheads degrade performance since most of the execution time is spent on creating and committing transactions-in extreme cases even more than 70\% [Chung et al. 2008]

Until now researchers have been trying to decrease STM overheads by applying various compiler and runtime optimizations on read and write barriers [Wang et al. 2007; Wu et al. 2009; Dragojevic et al. 2009; Afek et al. 2011], or by accelerating some STM functionalities in hardware [Stipic et al. 2012; Saha et al. 2006; Minh et al. 2007; Shriraman et al. 2007]. In our work, we follow a different approach and focus on reducing the overheads of starting and committing transactions.

This paper makes the following contributions:

-We introduce Transaction Coalescing (TC), a profile-guided compiler technique that lowers the overheads of starting and committing transactions. TC merges two or more consecutive transactions into a large transaction that has less transactional overhead compared to the original unmerged transactions (we use "merging" and "coalescing" interchangeably). To the best of our knowledge, this is the first profileguided compiler optimization technique for STM systems.

-We develop a profiling tool for collecting and analysing characteristics of a transactional application.

-We design a transaction coalescing heuristic that identifies candidate transactions suitable for merging.

-We implement TC as a profile-guided compiler optimization that uses profile information and the transaction coalescing heuristic to merge the candidate transactions.

We evaluate our proposal using the STAMP [Minh et al. 2008], red-black tree, and hash table benchmarks where we show profiling information for all the benchmarks. Using the profile-guided optimization, we identify the benchmarks that benefit from TC, and for these benchmarks, we show full statistics and performance improvement gained by applying TC. TC improves the performance of hash table significantly and the performance of Vacation and SSCA2 benchmarks by $19.4 \%$ and $36.4 \%$, respectively, when running with 12 threads.

The reminder of this article is organized as follows: In Section 2, we present transactional overheads as motivation of our work. In Section 3, we introduce transaction coalescing, a novel profile-guided compiler technique. In Section 4, we present our profiling tool, transaction coalescing heuristic, and explain how a compiler uses the heuristic for transaction coalescing. In Section 5, we show experimental results. Section 6 compares our work to previous studies. Finally, we conclude in Section 7.

\section{TRANSACTIONAL OVERHEADS}

A programmer writes a TM application by identifying sections of code for atomic execution isolated from other threads, and puts the code in tm_atomic blocks, i.e. transactions. A compiler and a runtime system ensure atomicity and isolation of transactions. At the beginning of a transaction, the compiler inserts a TX_START() function call. TX_START() initializes the transactional metadata and creates the snapshot of the register file and local variables. The compiler instruments all read and write operations that access shared memory locations. The functions TX_READ() and TX_WRITE() keep track of all speculative reads and writes. At the end of the transaction, the compiler inserts a TX_COMMIT () function call to make speculative writes globally visible.

In order to determine the overheads of TX_START() and TX_COMMIT () functions, we implement a very simple interest rate calculation benchmark ${ }^{1}$ (Figure 1(a)) where each thread calculates account balance for several accounts within a transaction denoted

\footnotetext{
${ }^{1}$ The benchmark resembles the main loop in transaction processing applications.
} 


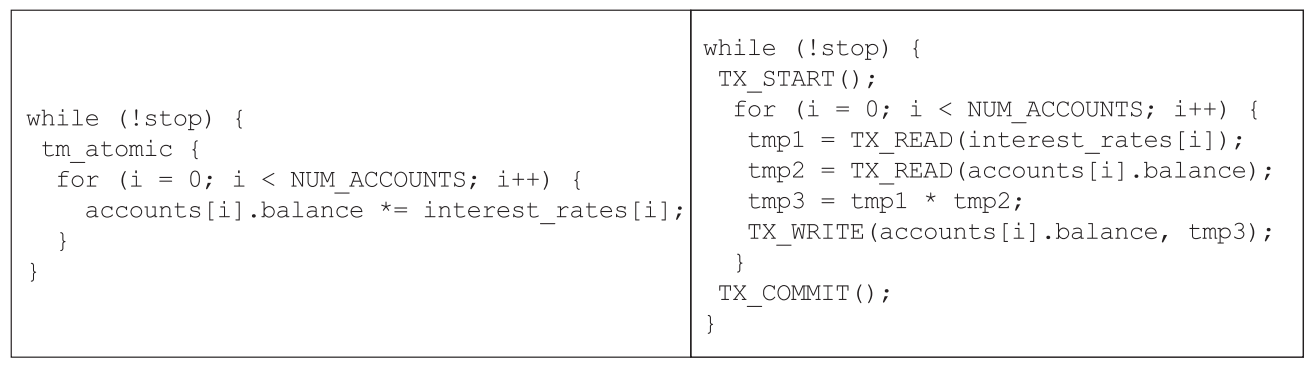

(a) transactional source code

(b) compiler-instrumented code

Fig. 1. Calculating account balance.

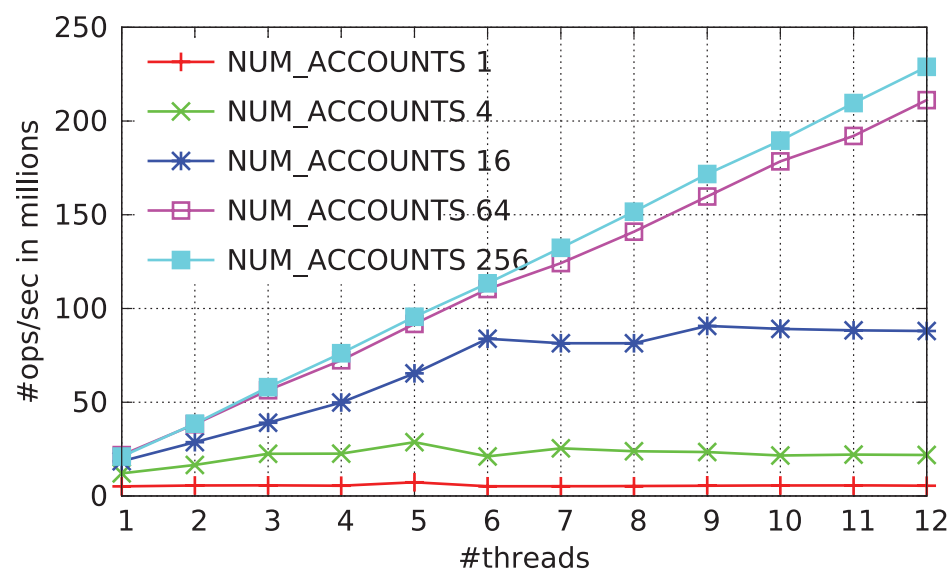

Fig 2. Throughput for the code from Figure 1. NUM_ACCOUNTS represents the number of balance calculations in a transaction. \#ops/sec represents the number of balance calculations per second.

with tm_atomic. Figure 1(b) shows the same code after transactional instrumentation. To measure the throughput, we use the environment described in Section 5, and we vary the NUM_ACCOUNTS parameter from 1 to 256 and the number of threads from 1 to 12 (Figure 2). We observe the following:

-As the NUM_ACCOUNTS parameter increases, the throughput increases as well. This happens because the total time spent in TX_START() and TX_COMMIT() decreases compared to the time spent inside the transaction.

-Increasing the transaction size is beneficial up to some limit. In our example, the throughput is very simmilar for NUM_ACCOUNTS $=64$ and NUM_ACCOUNTS $=256$. The further increase of the NUM_ACCOUNTS parameter gives negligible performance improvements because the time spent in TX_START() and TX_COMMIT() is small with respect to the transaction size.

- The biggest jump in the throughput is for the small values of NUM_ACCOUNTS parameter (NUM_ACCOUNTS $=4$ or NUM_ACCOUNTS $=16$ ). This shows that reducing the TX_START () and TX_COMMIT() overheads has a big performance improvement for small transactions.

-For small transactions (NUM_ACCOUNTS $<=16$ ), the throughput is limited when transactions execute on different physical sockets ${ }^{2}$ (even though the threads operate on

${ }^{2}$ The throughput is limited to 6 cores because we use two socket machine where each socket has 6 cores on chip. 


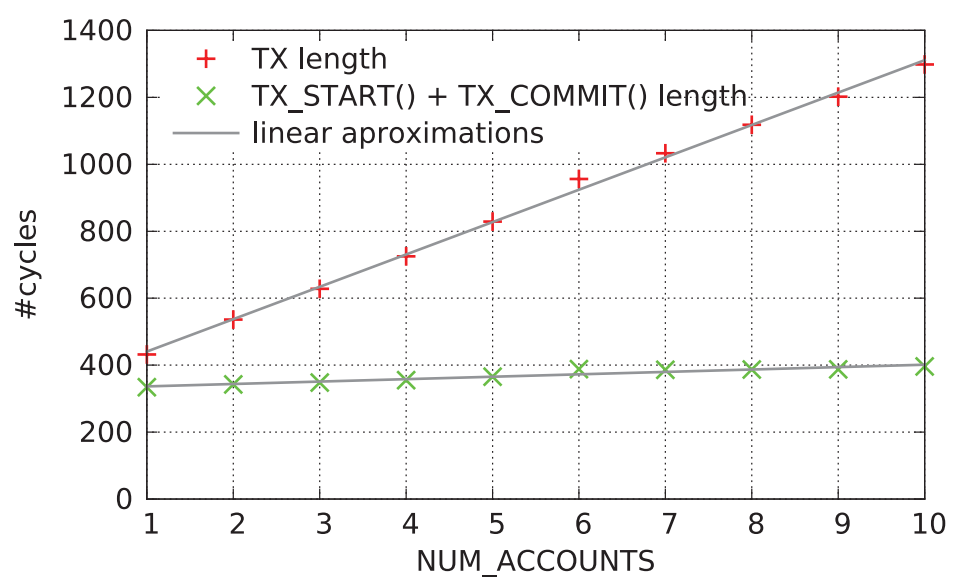

Fig. 3. Execution time in cycles for the code from Figure 1 when running with 1 thread.

independent data). The throughput is limited because STM serializes transactions at commit time by updating the shared global "commit counter," and small transactions finish before the "commit counter" gets in the exclusive state in CPU's private L1 cache. For larger transactions (NUM_ACCOUNTS > 16), the transactions execution time is larger than time required to get the "commit counter" in exclusive state (the transaction execution is overlapped with internal STM synchronization).

From this simple example, we conclude that increasing the size of transactions up to some limit reduces TX_START() and TX_COMMIT() overheads. The new insight is that we have to pay the price of transaction initialization just once if we join two or more transactions. So, we remove some of the cost of the transaction initialization.

In order to quantify the transactional overheads of TX_START() and TX_COMMIT(), we execute the interest rate calculation benchmark with 1 running thread. Figure 3 shows that TX_START () and TX_COMMIT() consume the largest part of a transaction when NUM_ACCOUNTS $=1$ (334 out of 432 cycles, which is $\left.77.3 \%{ }^{3}\right)$. Increasing the NUM_ACCOUNTS decreases the percentage of the transactional overheads compared to the whole transaction execution. Therefore, we conclude that short transactions are preferable for reducing the transactional overheads.

\section{TRANSACTION COALESCING}

In this article, we introduce Transaction Coalescing (TC), a compiler technique that merges small transactions with large transactional overhead into a large transaction with lower transactional overhead. TC merges two or more consecutive transactions or transactions separated by nontransactional code.

In Section 2, we showed that for small transactions increasing the number of operations per transaction reduces the transactional overhead of TX_START() and TX_COMMIT(). In this section, we provide a few use cases for applying TC and reducing the transactional overhead: (i) transactions are consecutive, (ii) a compiler applies traditional compiler optimizations (loop unrolling and function inlining), and (iii) transactions are separated by nontransactional code.

Figure 4(a) shows an example of incrementing two counters in two transactions. The compiler transforms the tm_atomic blocks and instruments the code inside. TC merges two consecutive transactions by removing TX_COMMIT() from the first transaction and

\footnotetext{
${ }^{3}$ The transactional overhead depends on the specifics of the STM implementation.
} 
transactional source code

after compiler transformations

after applying TC

\begin{tabular}{|c|c|c|}
\hline $\begin{array}{l}\text { tm_atomic }\{ \\
\text { counter } 1++;\end{array}$ & {$\left[\begin{array}{l}\text { TX_START (); } \\
\text { tmp1 = TX_READ (counter } 1) ; \\
\text { tmp1++; } \\
\text { TX_WRITE (counter1, tmp1) ; } \\
\text { TX_COMMIT (); }\end{array}\right.$} & {$\left[\begin{array}{l}\text { TX_START (); } \\
\text { tmp1 = TX_READ (counter } 1) ; \\
\text { tmp1++; } \\
\text { TX_WRITE (counter } 1, \text { tmp } 1) ; \\
\text { // no TX_COMMIT (); }\end{array}\right.$} \\
\hline $\begin{array}{l}\text { tm_atomic }\{ \\
\text { counter } 2++;\end{array}$ & {$\left[\begin{array}{l}\text { TX_START (); } \\
\text { tmp2 = TX_READ (counter } 2) ; \\
\text { tmp2++; } \\
\text { TX_WRITE (counter } 2, \operatorname{tmp} 2) ; \\
\text { TX_COMMIT (); }\end{array}\right.$} & $\begin{array}{l}\text { I/ no TX_START (); } \\
\text { tmp2 = TX_READ (counter2); } \\
\text { tmp2++; } \\
\text { TX_WRITE (counter2, tmp2); } \\
\text { TX_COMMIT (); }\end{array}$ \\
\hline
\end{tabular}

(a) The basic example of TC

\begin{tabular}{|c|c|c|}
\hline $\begin{array}{l}\text { for }(i=0 ; i<1000 ; i++)\{ \\
\quad \text { tm_atomic }\{ \\
\quad \text { a }[i]++;\end{array}$ & $\begin{array}{l}\text { for }(i=0 ; i<1000 ; i+=2)\{ \\
\quad \text { tm_atomic }\{ \\
\quad \text { a }[i]++; \\
\} \\
\text { tm_atomic }\{ \\
\quad \text { a }[i+1]++; \\
\}\end{array}$ & $\begin{array}{l}\text { for }(i=0 ; i<1000 ; i+=2)\{ \\
\quad \begin{array}{l}\text { tm_atomic }\{ \\
\quad a[i]++; \\
\quad a[i+1]++; \\
\}\end{array}\end{array}$ \\
\hline
\end{tabular}

(b) Loop unrolling and TC

\begin{tabular}{|c|c|c|}
\hline $\begin{array}{l}\text { void update_counters () \{ } \\
\text { increment (); } \\
\text { decrement (); } \\
\text { \} inline void increment () \{ } \\
\text { tm_atomic \{ } \\
\text { counter } 1++; \\
\text { \} } \\
\text { inline void decrement () \{ } \\
\text { tm_atomic \{ } \\
\text { counter } 2--; \\
\text { \} }\end{array}$ & $\begin{array}{l}\text { void update_counters () } \\
\text { tm_atomic }\{ \\
\text { counter } 1++; \\
\} \\
\text { tm_atomic }\{ \\
\text { counter } 2--; \\
\}\end{array}$ & $\begin{array}{l}\text { void update_counters () \{ } \\
\text { tm_atomic }\{ \\
\text { counter } 1++; \\
\text { counter } 2--; \\
\}\end{array}$ \\
\hline
\end{tabular}

(c) Function inlining and TC

\begin{tabular}{|c|c|c|}
\hline $\begin{array}{l}\text { void work_with_list() \{ } \\
\text { int el = get_elem(); } \\
\text { process(el); } \\
\text { put_elem(el); } \\
\text { \} } \\
\text { int get_elem()\{ } \\
\text { int el; } \\
\text { tm_atomic \{ } \\
\quad \text { el = list.pop (); } \\
\text { \} return el; } \\
\text { \} void put_elem(int el) \{ } \\
\text { tm_atomic \{ } \\
\text { \} list.push (el); } \\
\text { \} }\end{array}$ & $\begin{array}{l}\text { void work_with_list() } \\
\text { int el; } \\
\text { tm_atomic }\{ \\
\quad \text { el = list.pop (); } \\
\text { \} } \\
\text { process }(e l) ; \\
\text { tm_atomic }\{ \\
\text { list.push }(e l) ; \\
\}\end{array}$ & $\begin{array}{l}\text { void work_with_list() } \\
\text { int el; } \\
\text { tm_atomic }\{ \\
\quad \text { el = list.pop(); } \\
\text { process (el); } \\
\text { list.push (el); } \\
\text { \} }\end{array}$ \\
\hline
\end{tabular}

(d) Code between transactions included in a merged transaction

Fig. 4. Compiler transformations: instrumentation and TC (a), optimizations and TC (b), (c), (d). In all examples the compiler inserts TX_START (), TX_COMMIT (), TX_READ (), TX_WRITE() calls like in (a); however, we do not show them in (b), (c), and (d) for clarity of code. 
TX_START() from the second transaction. This reduces the overall overhead associated with the transactional execution.

Figure 4(b) illustrates an example where the compiler uses TC after loop unrolling. The for loop atomically increments each element of the array, and each iteration of the loop pays the overhead for TX_START() and TX_COMMIT(). After the loop unrolling, two consecutive increments can be coalesced in one large transaction with lower transactional overhead.

Figure 4(c) demonstrates how TC can be combined with function inlining. The figure shows two transactions in two separate functions (increment () and decrement ()). After inlining these functions, two transactions become consecutive, and TC can be applied. TC optimization pass depends on unrolling and inlining the same way, for example, modulo scheduling (also known as software pipelining) depends on loop unrolling (modulo scheduling requires loop unrolling pass)

Until now, we have considered the ideal cases when transactions are consecutive. However, a more likely case is that a piece of code separates transactions. For example, Figure 4(d) shows typical operations with a shared list: the function work_with_list() atomically pops an element from the list, processes the element outside of tm_atomic blocks, and atomically pushes the processed element to the list. The compiler inlines the functions get_elem() and put_elem() and now TC creates one transaction with pop (), push (), and a transactional version of process (). The instrumentation of the process () function introduces an additional overhead. Therefore, to find transactions for merging, we need to analyze both the code within transactions (because of the benefit of TC) and the code between transactions (because of the disadvantages of TC).

\section{APPLYING TC}

In this section, we introduce our profiling tool, transaction coalescing heuristic, and compiler pass. The tool generates profile information, the heuristic uses the profile information to find candidate transactions, and the compiler pass merges the candidate transactions.

\subsection{Profiling Tool}

We developed a profiling tool for finding transactions that benefit from TC. The tool executes an unmodified program, and gathers statistics from a single-threaded execution. The tool outputs the following information:

-Transaction execution information. For every transaction, the tool collects (i) the number of cycles executed in the transaction, (ii) the percentage of the total execution time spent in the transaction, and (iii) the sizes of read and write sets. The tool uses the transaction coalescing heuristic to identify small and frequently executed transactions that are candidates for TC.

- Transaction transition information. For every pair of transactions the tool calculates a transaction transition matrix and a transaction distance matrix. The transaction transition matrix counts the number of transitions between transactions, and the transaction distance matrix calculates the average number of cycles executed between transactions. The tool uses the transaction coalescing heuristic to find transactions that are close to each other during the execution.

To use the profiling tool, we compile and run STAMP benchmarks (using the input parameters from Table I) and two micro-benchmarks (hash table and red-black tree). We show the transaction execution information in Table II and the transaction transition information in Table III and Table IV. Although some benchmarks have more than 3 transactions, we present only the transactions that occupy most of the benchmark execution time. 
Table I. STAMP Benchmark Input Parameters

\begin{tabular}{|l|l|}
\hline & benchmark input arguments \\
\hline Bayes & -v32 -r4096 -n10 -p40 -i2 -e8 -s1 \\
\hline Genome & -g16384 -s64 -n16777216 \\
\hline Intruder & $-\mathrm{a} 10-\mathrm{l} 128$-n262144 -s1 \\
\hline Kmeans & -m40 -n40 -t0.00001 -i rnd-n65536-d32-c16 \\
\hline Labyrinth & -i rnd-x128-y128-z5-n128.txt \\
\hline SSCA2 & -s20 -i1.0 - u1.0 -13 -p3 \\
\hline Vacation & -n4 -q60 -u90 -r1048576 -t4194304 \\
\hline Yada & -a15 -i ttimeu1000000.2 \\
\hline
\end{tabular}

Table II. Transaction Execution Information

The length is the average number of cycles spent in a transaction. The execution time of a transaction is the average percentage of total execution time spent in the benchmark. The read/write set size is the average size of the read/write sets of a transaction. Gray cells show small transactions, dark gray show frequent transactions, light gray cells show transactions with small read and write sets, and circled cells show candidate transactions for TC.

\begin{tabular}{|c|c|c|c|c|c|c|}
\hline & $\operatorname{tx} 1$ & tx2 & tx3 & tx1 & tx2 & tx3 \\
\hline benchmark & \multicolumn{3}{|c|}{$\overline{\text { Bayes }}$} & \multicolumn{3}{|c|}{ Labyrinth } \\
\hline length (cycles) & $908,161,356$ & $536,427,523$ & $406,716,287$ & $110,363,661$ & 262,308 & 2,735 \\
\hline execution time & $46.31 \%$ & $27.36 \%$ & $20.74 \%$ & $99.74 \%$ & $0.24 \%$ & $<0.1 \%$ \\
\hline read set size & 11509169.82 & 6465018.77 & 5057245.93 & 2751731.61 & 7.96 & 2.00 \\
\hline write set size & 2344162.23 & 1309395.11 & 1025313.10 & 335355.95 & 0.99 & 4.00 \\
\hline benchmark & \multicolumn{3}{|c|}{ Genome } & \multicolumn{3}{|c|}{ Rb tree } \\
\hline length (cycles) & 20,789 & 14,640 & 960 & 980 & 2,016 & 2,153 \\
\hline execution time & $65.42 \%$ & $33.88 \%$ & $<0.1 \%$ & $90.11 \%$ & $1.6 \%$ & $0.68 \%$ \\
\hline read set size & 186.01 & 137.10 & 3.83 & 29.82 & 31.75 & 38.86 \\
\hline write set size & 12.07 & 4.00 & 3.78 & 0.00 & 7.00 & 9.00 \\
\hline benchmark & \multicolumn{3}{|c|}{ Hash table } & \multicolumn{3}{|c|}{ SSCA2 } \\
\hline length (cycles) & & 664 & 921 & & $14,836,229$ & 6,386 \\
\hline execution time & $78.53 \%$ & $1.12 \%$ & $0.86 \%$ & $59.31 \%$ & $<0.1 \%$ & $<0.1 \%$ \\
\hline read set size & 2.50 & 3.00 & 4.00 & 5.00 & 1.00 & 1.00 \\
\hline write set size & 0.00 & 1.66 & 4.00 & 2.00 & 1.00 & 1.00 \\
\hline benchmark & \multicolumn{3}{|c|}{ Intruder } & \multicolumn{3}{|c|}{ Vacation } \\
\hline length (cycles) & 14,933 & 662 & 579 & & 6,595 & 1,897 \\
\hline execution time & $81.23 \%$ & $3.6 \%$ & $3.15 \%$ & $71.01 \%$ & $15.0 \%$ & $4.27 \%$ \\
\hline read set size & 163.84 & 6.00 & 4.13 & 9.21 & 84.90 & 9.00 \\
\hline write set size & 13.85 & 1.00 & 1.13 & 1.00 & 6.99 & 1.00 \\
\hline benchmark & \multicolumn{3}{|c|}{ Kmeans } & \multicolumn{3}{|c|}{ Yada } \\
\hline length (cycles) & 6,199 & 465 & 937 & 379,700 & 1,633 & 1,224 \\
\hline execution time & $47.46 \%$ & $1.19 \%$ & $<0.1 \%$ & $98.66 \%$ & $0.58 \%$ & $0.32 \%$ \\
\hline read set size & 130.00 & 1.00 & 1.00 & 7264.59 & 14.98 & 11.70 \\
\hline write set size & 33.00 & 1.00 & 1.00 & 1468.79 & 2.20 & 1.54 \\
\hline
\end{tabular}

Table III. Transaction Transition Matrix: The Probability of Transitions between Transactions For candidate transactions "tx1" from Table II, the dominant transition is to the transactions "tx1" (circled cells).

\begin{tabular}{|c|c|c|c|c|c|c|c|c|c|c|c|}
\hline Ht. & tx1 & tx2 & tx3 & Vac. & tx1 & tx2 & $\mathrm{tx} 3$ & SSCA2 & tx1 & $\mathrm{tx} 2$ & tx3 \\
\hline tx1 & $98.76 \%$ & $0.85 \%$ & $0.38 \%$ & tx1 & $89.98 \%$ & $\overline{5.02 \%}$ & $4.99 \%$ & tx1 & $99.99 \%$ & - & $<0.01 \%$ \\
\hline tx2 & $92.97 \%$ & - & $7.03 \%$ & tx2 & $89.98 \%$ & $5.18 \%$ & $4.83 \%$ & $\mathrm{tx} 2$ & 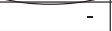 & - & \\
\hline tx3 & $100 \%$ & 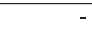 & - & tx3 & $90.53 \%$ & $4.81 \%$ & $4.66 \%$ & tx3 & $<0.01 \%$ & & $99.99 \%$ \\
\hline
\end{tabular}


Table IV. Transaction Distance Matrix: The Average Number of Cycles between Transactions Circled cells confirm candidate transactions suitable for TC.

\begin{tabular}{|r||r|r|r|r||r|r|r||r||r|r|r|}
\hline Ht. & tx1 & tx2 & tx3 \\
\hline \hline tx1 & Vac. & tx1 & tx2 & tx3 & 97 & 90 \\
\hline tx2 & 110 & - & 112 \\
\hline tx1 & 219 & 222 & 230 & SSCA2 & tx1 & tx2 & tx3 \\
\hline tx3 & 109 & - & - \\
\hline$t x 2$ & 214 & 220 & 231 & tx2 & - & - & - \\
\hline tx3 & 178 & 177 & 189 & tx3 & $138 \mathrm{~K}$ & - & 74 \\
\hline
\end{tabular}

\subsection{Transaction Coalescing Heuristic}

The transaction coalescing heuristic uses profile generated statistics to identify candidate transactions suitable for merging. The heuristic looks for transactions that are small, frequent, and close to each other, by using the following rules:

-A small transaction is a transaction that executes in fewer than 3,000 cycles, on average, ${ }^{4}$ and has fewer than 16 and 4 elements in read and write sets, ${ }^{5}$ respectively. -A frequently executed transaction is a transaction that consumes at least $10 \%$ of the execution time of the program.

- Close transactions are a pair of transactions that have the transition probability higher than $80 \%$ and have fewer than 1,000 cycles between each other on average. ${ }^{6}$

The information from Table II is crucial to find small and frequently executed transactions that are candidates for TC. The heuristic identifies that hash table, Vacation, and SSCA2 have candidate transactions suitable for TC (circled cells in the table). For example, the transaction "tx1" of Vacation executes in 1,742 cycles and consumes $71.01 \%$ of the total execution time. However, transactions not suitable for TC are either (i) large transactions (e.g., "tx1" in Bayes), or (ii) infrequently executed transactions (e.g., "tx2" in Intruder).

Table III shows the transaction transition matrix for the three benchmarks (hash table, Vacation, and SSCA2) where TC can be applied. For all these benchmarks, the dominant transition is from "tx1" to "tx1" with more than $80 \%$ transition probability. Thus, "tx1" becomes a stronger candidate for TC and the heuristic has to ensure that multiple invocations of "tx 1 " are close to each other. The heuristic uses the transaction distance matrix (Table IV) to get the distance in cycles between the invocations of "tx1." Finally, the heuristic confirms that in all the cases, intertransaction distance is fewer than 1,000 cycles (circled cells), thus the transactions are close.

Based on the heuristic, the compiler applies TC on "tx1" of hash table, Vacation, and SSCA2. This implies that repetitive invocations of "tx1" should be coalesced. Therefore, the compiler unrolls the loop where " $\mathrm{x} x 1$ " is located and applies TC on multiple instances of "tx1."

\subsection{Compiler Pass}

We implement TC as a profile-guided optimization pass using Berkeley's C Intermediate Language (CIL) tool [Necula et al. 2002]. CIL is a compiler frontend that transforms C language constructs to CIL's Abstract Syntax Tree (AST) and uses GCC [GCC 2013] as a backend compiler. We implement the transaction coalescing algorithm in CIL where the algorithm merges transactions identified by the transaction coalescing heuristic.

\footnotetext{
${ }^{4}$ We choose 3,000 cycles as the upper limit because in this case TX_START() and TX_COMMIT() create overhead of more than $10 \%$ of transaction execution time (Section 2 shows that the overhead is about 300 cycles).

${ }^{5}$ These sizes of read and write sets work well in practice.

${ }^{6}$ The upper limit of 1,000 cycles between transactions is determined empirically.
} 


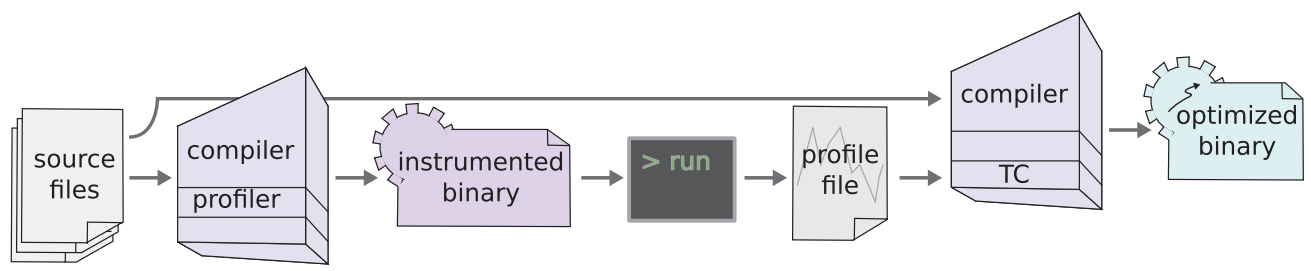

Fig. 5. The workflow of transaction coalescing. A user compiles source files with the profiler and runs the application to get a profile file. The user then compiles the source files again when TC coalesces transactions according to the profile file.

In Figure 5, we show the workflow of our implementation. The user compiles an application with profiling enabled and runs the application ${ }^{7}$ to generate the profile information. The second time the user compiles the application, the TC compiler applies the transaction coalescing heuristic on the profile information to identify candidate transactions. TC is dependant on loop unrolling and function inlining, which gives the compiler opportunity to coalesce the candidate transactions using the transaction coalescing algorithm. In our implementation, the compiling and running steps are totally automatized.

Transaction coalescing algorithm merges two transactions at the AST level (Figure 6) into one large transaction. ${ }^{8}$

The algorithm executes in three steps:

(1) The algorithm identifies transactions (TX1 and TX2) selected for merging (Figure 6(a)).

(2) The algorithm finds the first common parent node $(f())$ of the transactions in the AST, identifies parent's children nodes (TX1 and if) that contain selected transactions, and creates a new transaction node (TX_coalesced) including these nodes and all their sibling nodes in between (TX1, <code3>, and if) (Figure 6(b)).

(3) The algorithm removes the original transaction nodes (TX1 and TX2) from the AST (Figure 6(c)).

\subsection{Transaction Coalescing-Correctness}

The transaction coalescing algorithm preserves atomicity and isolation of transactions. In other words, in the case of race free programs, the coalescing preserves the correctness of a program and it is safe to merge two adjacent transactions ${ }^{9}$ or to include nontransactional code in a transaction. When nontransactional code becomes part of a larger transaction, the transaction coalescing algorithm uses the compiler to instrument the accesses to shared data. We would like to emphasise that the compiler will not instrument the accesses to thread-local variables. To illustrate this, we use the following example:

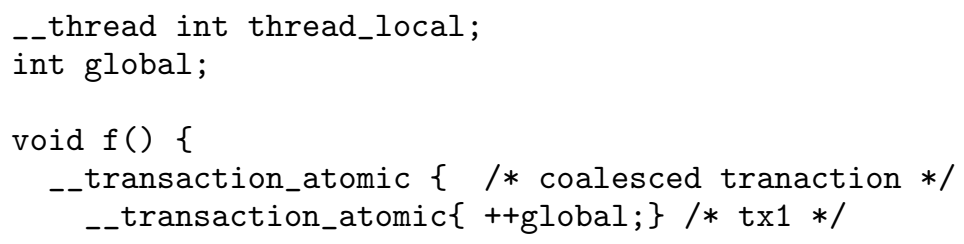

\footnotetext{
${ }^{7}$ Because transaction coalescing is a profile-guided optimization technique, the user has to provide a representative input set for the sample run.

${ }^{8}$ The algorithm can be used recursively to merge more than two transactions.

${ }^{9}$ The TC algorithm is analogous to the lock coalescing algorithm [Diniz and Rinard 1997].
} 

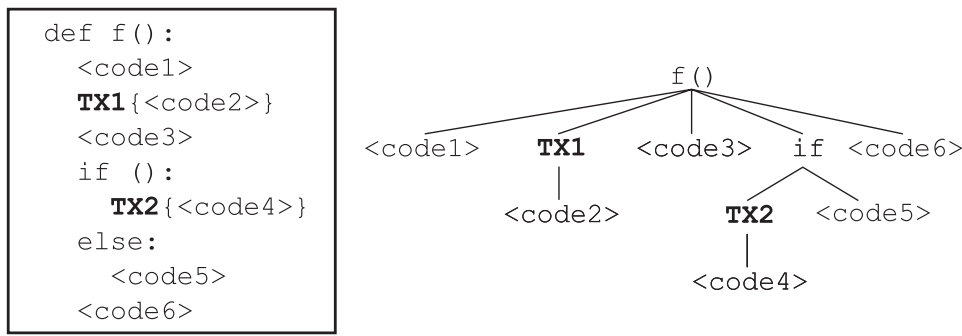

(a) Transactions identified
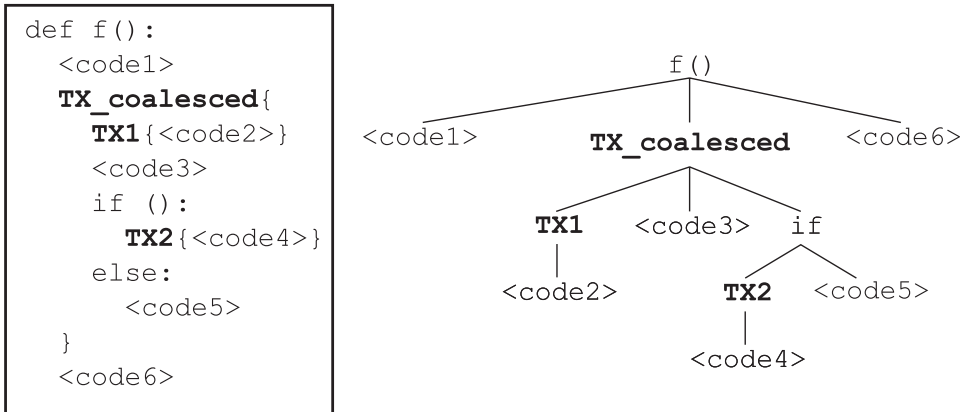

(b) Transactions coalesced

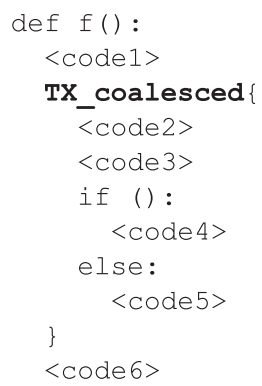

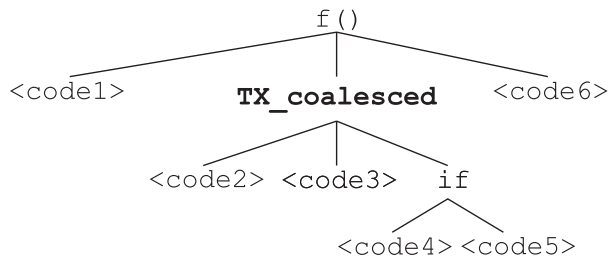

(c) Transactions flattened

Fig. 6. Transaction coalescing algorithm for merging transactions at the Abstract Syntax Tree (AST) level. The AST on the right is equivalent to the source code on the left.

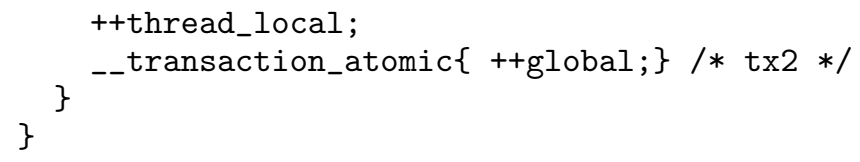

The example shows the function $f()$ that has one coalesced transaction. The coalesced transaction includes two smaller transactions (tx1 and tx2) and nontransactional code (++thread_local). The following code shows the disassembly of the function $f()$ :

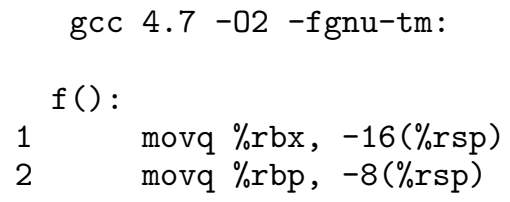




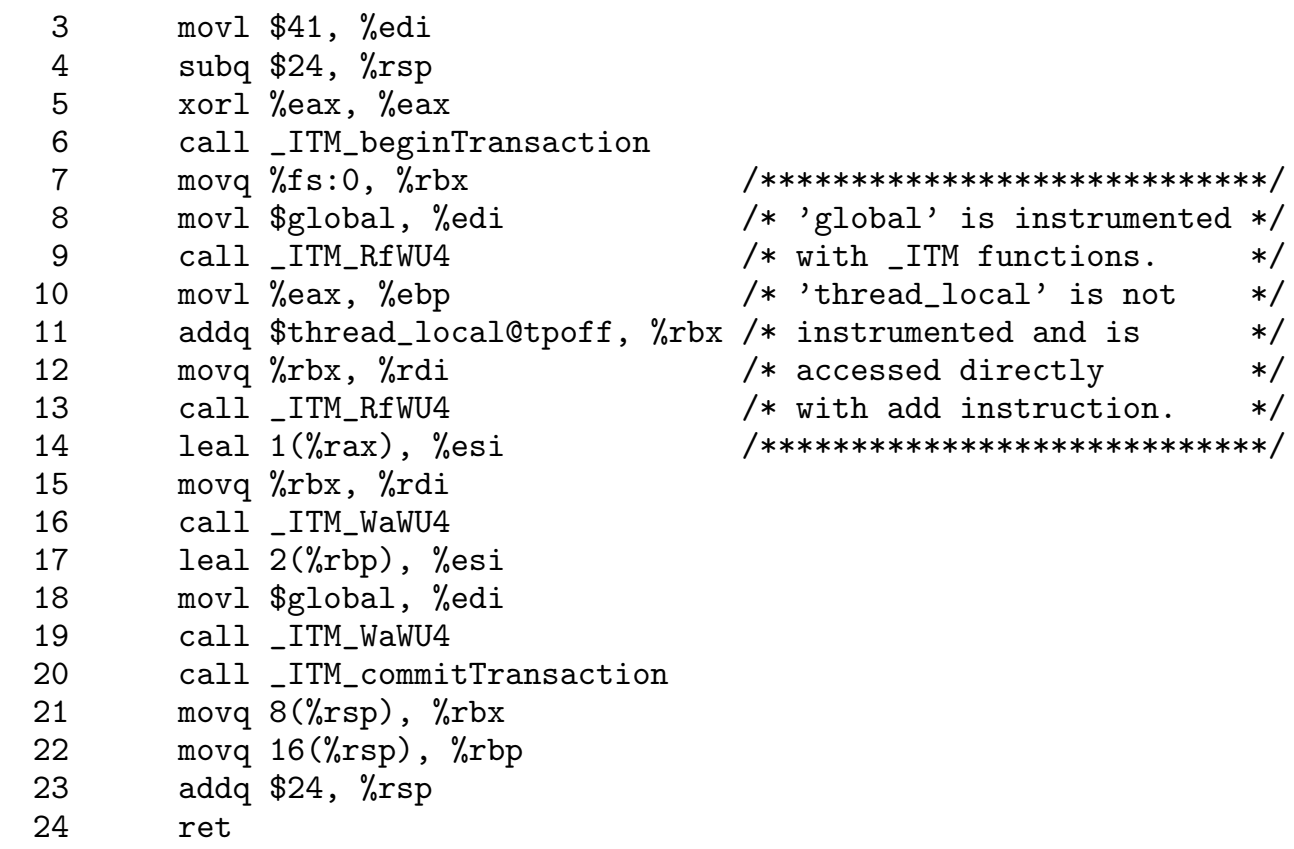

The TC algorithm relies on the ability of the compiler to instrument only accesses to shared variables (global) but not accesses to thread-local variables (thread_local, line 11). If a transaction aborts, the transaction restores variables as they were at the beginning of the transaction, which preserves the semantics of the original program.

GCC provides proper instrumentation of thread-local variables in the following way: Before the start of a transaction the current values of the thread-local variables that are accessed inside of a transaction are pushed on the stack. During the transaction execution, the accesses to thread-local variables are not instrumented. Just in the case of the abort, the values of thread-local variables are restored from the stack.

\subsection{Executing Non-undoable Code}

It is possible that the transaction coalescing algorithm creates a coalesced transaction that includes non-undoable operations (e.g. asm, volatile, printf()). In that case, the compiler detects the non-undoable code and executes the transaction guaranteeing that it will commit without any abort. The more elaborate explanation follows.

Sometimes, the memory accesses (in the nontransactional code) are aliased to MMIO space. The common programming practice is to access MMIO addresses with ACCESS_ONCE () macro defined as:

\#define ACCESS_ONCE $(\mathrm{x})$ (*(volatile typeof $(\mathrm{x}) *) \&(\mathrm{x}))$ \}

GCC treats all accesses to volatile variables, all system calls, and all non-transactified function calls as non-undoable code. At compile time, GCC has the access to all transactional code in the program. If any code path in the transaction tries to execute non-undoable code, the compiler inserts the code that acquires the shared global lock. The first transaction that acquires the shared global lock validates itself. The validation can succeed or fail:

(1) If the validation succeeds, the transaction commits its speculative changes and continues executing while holding the shared global lock. When the transaction ends, it releases the shared global lock. 
(2) If the validation fails, the transaction rollbacks and restarts without releasing the global shared lock. Now, the transaction runs from the beginning to the end while holding the shared global lock. When the transaction ends, it releases the shared global lock.

In both cases, the execution of non-undoable code is protected with the shared global lock, which ensures correct execution of non-undoable transactions (no transaction can abort the transaction holding the shared global lock). Other transaction that tries to execute non-undoable code will be blocked on the acquisition of the shared global lock.

If the program is not race free, including nontransactional code in a transaction can introduce deviation of the program execution in the presence of rollbacks. ${ }^{10}$ The compiler has to ensure that nontransactional code included in the transactions can not be accesses by other threads concurrently. At this point in time, TC is an unsafe optimization and a programmer should enable TC explicitly only if he/she knows that the program is race free.

In Section 5, we evaluate the performance improvements of TC on the benchmarks selected for transaction coalescing.

\section{EVALUATION}

We perform experiments on a Sun Fire x4140 system equipped with two Six-Core AMD Opteron 2427 (12 cores in total), 32GiB RAM, running Linux 2.6.32-5. We compile the applications with GCC 4.7 with Transactional Memory support and link with TinySTM [Felber et al. 2008] 1.0.3. We implement the TC compiler pass in CIL 1.5 and use GCC as a backend. In this section, we explain the implementation of the applications we selected in Section 4 (i.e., hash table, Vacation, and SSCA2), and we evaluate their performance.

\subsection{Benchmarks}

Hash table executes repetitively the transactional lookup (), add (), or remove () functions. Each function operates on shared data stored in the hash table. We control the number of updates (add () and remove ()) relative to the total number of operations (lookup (), add (), and remove ()) with the update rate parameter. We include a function work() between the invocations of remove() and add() to show how nontransactional code instrumentation influences TC. work() executes a simple mathematical function $(f(x)=13 * x \bmod y)$ work loop count (wlc) times.

Vacation from the STAMP benchmark suite implements a travel reservation system powered by an in-memory database. Several client threads interact with the database through transactional requests. The performance is measured as the number of served client requests per second. Zyulkyarov et al. [2010] shows that the performance bottleneck in Vacation is the red-black tree that is used as database storage, and they suggest replacing it with a hash table. We follow the same approach. Moreover, we identified another performance bottleneck: a function for generating random numbers was on the critical path limiting the throughput. We moved the generation of random numbers to the initialization part of the benchmark, where we populate per thread arrays with random numbers. We replaced the original random number function with an array indexing function that reads the random numbers from the arrays. In this way, we improved the throughput of the original benchmark without modifying its semantics.

SSCA2 [Bader and Madduri 2005] is a synthetic benchmark that operates on a large, directed, weighted multigraph. The main loop of SSCA2 traverses all the edges

\footnotetext{
${ }^{10}$ STAMP benchmarks suite is race free because all the shared variables are accessed only inside of transactions and TC can be applied safely.
} 
of the graph. The graph can be traversed in any order and the final execution of the benchmark is the same. Thus, transactions in the main loop execute in arbitrary order. We modified the main loop, and instead of executing the transactions immediately, we buffer the values of the variables used in the transactions. When the buffer gets full, we execute all the transactions in a tight loop.

Applying TC on the benchmarks: In Section 4.2, the compiler identified "tx1" of hash table, Vacation, and SSCA2 as a candidate transaction for TC. To merge "tx1" with itself, TC unrolls the loop where "tx1" is located and merges two (or more) instances of "tx1" into one transaction. ${ }^{11}$ The number of coalesced transactions in the TC compiler pass is controlled by the aggressive flag (i.e., when aggressive $=\mathrm{n}$, the compiler pass merges $\mathrm{n}$ transactions. If it is not possible to merge $\mathrm{n}$ transactions, the compiler finds a loop that contains transactions and unrolls it $n$ times. After loop unrolling, the compiler merges transactions. If the compiler does not find the loop that contains transactions suitable for merging, the compiler bails out. In our implementation, we use aggressive $=2$ as a default parameter, but in the benchmarks, we vary the aggressive factor between 2 and 16. In "a ggressive 2/4/8/16" the loop is unrolled more times to merge 2 , 4,8 , and 16 instances of "tx 1 ," respectively, into one transaction.

\subsection{Results}

All of the benchmarks (hash table, SSCA2, Vacation) initialize their input set using the random() function for which we provide the initial seed value. Hash table uses random() to generate the sequence of hash table update/lookup operations (insert 10, delete 3 , delete 9, lookup 17, insert 11, ..), SSCA2 uses random() to generate graph structure, and Vacation uses random() to populate in-memory database and to generate the sequence of accesses to the database (make_car_reservation 3, delete_customer 7, delete_flight_reservation $15, \ldots$. . For every run of the benchmarks we initialize the seed to a different value. By changing the seed values, the benchmarks generate one input set for profiling and different input set for measurement phase (which is necessary for obtaining correct results).

Figure 7 shows the performance of the hash table for various update rates $(1 \%, 5 \%$, and $20 \%)$, and work loop counts $(0,100$, and 1,000). We measure the performance as the number of million operations (lookup (), add (), or remove()) per second. TC improves the performance for all the variations of the input parameters. When transaction coalescing is too aggressive, the performance starts suffering, for example, "aggressive 16 " performs worse than "aggressive 8" in Figure 7(g). However, "aggressive 2" (the default aggressive factor) performs from $10.7 \%$ (Figure $7\left(\right.$ a)) to $158.5 \%{ }^{12}$ (Figure $7(\mathrm{~h})$ ) better than "original," when running with 12 threads. When the update rate is $20 \%$ (Figures 7(g), 7(h), and 7(i)) and the aggressive factor is 16, the number of aborts goes up to $90 \%$. For this particular benchmark, the high abort rate has small impact to scalability, but this cannot be stated in general. ${ }^{13}$

Figure 8 shows "original," "improved," and "aggressive" versions of Vacation benchmark. "Improved" is based on "original" where red-black tree is substituted with hashtable and where random-number generation is moved to the initialization part of the benchmark (explained in Section 5.1). These changes improved the performance of "original" significantly. We apply TC ("aggressive n") on the improved baseline ("improved") to show the benefit of using TC.

\footnotetext{
${ }^{11} \mathrm{TC}$ would fail in case of Vacation and SSCA2 if the compiler does not unroll loop and does not inline functions with transactions.

${ }^{12}$ The improvement of $158.5 \%$ is mostly due to bad scalability of the hash table with $20 \%$ update rate.

${ }^{13}$ The high abort rate is caused by transactions that repeatedly abort immediately at the beginning of a transaction. Effectively, the aborting transactions spins on the conflicting address.
} 

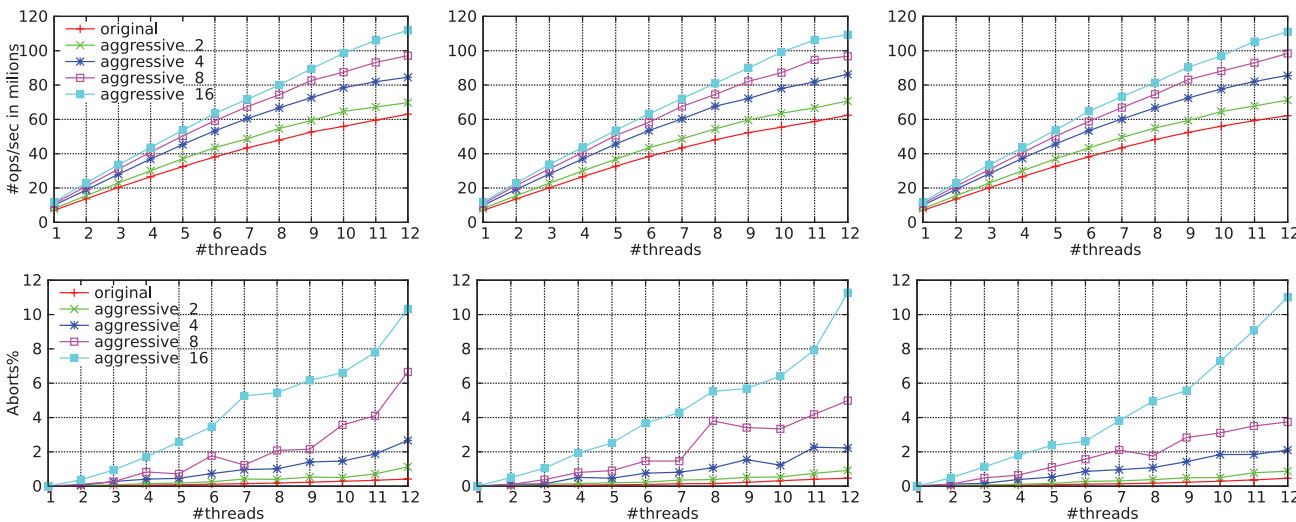

(a) $\mathrm{ur}=1 \%$; wlc $=0$

(b) ur $=1 \%$; wlc $=100$

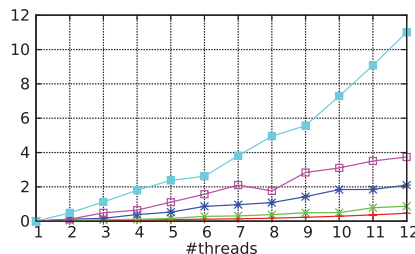

(c) $\operatorname{ur}=1 \% ;$ wlc $=1,000$
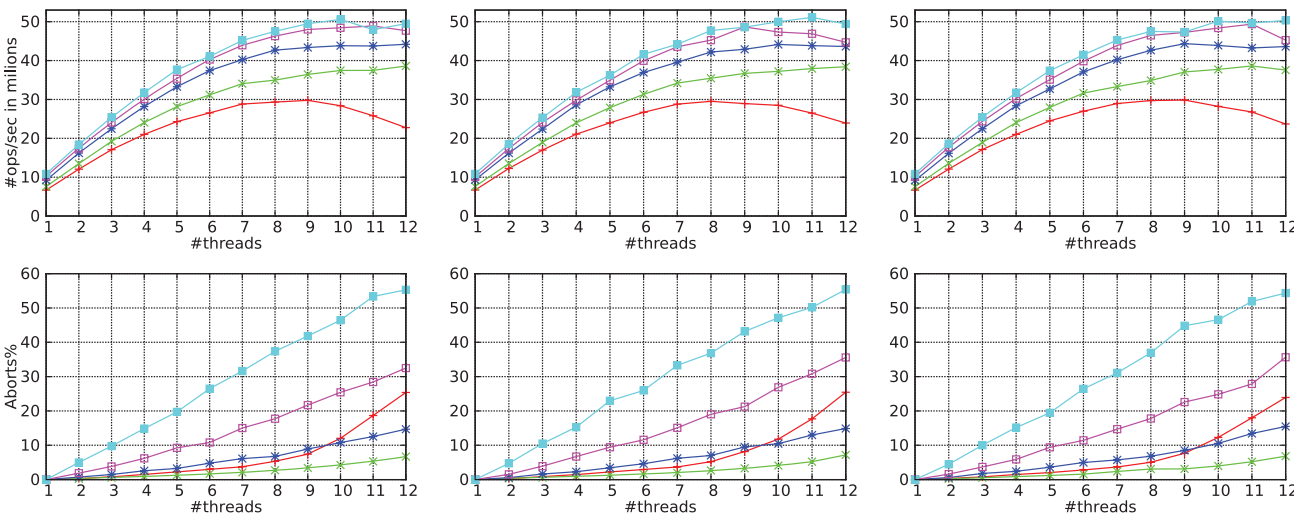

(d) $\mathrm{ur}=5 \%$; wlc $=0$

(e) $\mathrm{ur}=5 \%$; wlc $=100$

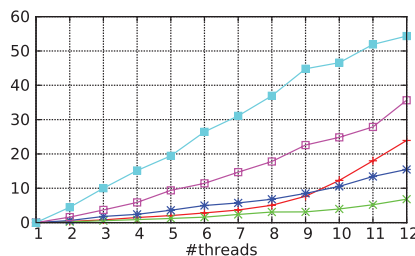

(f) $\mathrm{ur}=5 \% ;$ wlc $=1,000$
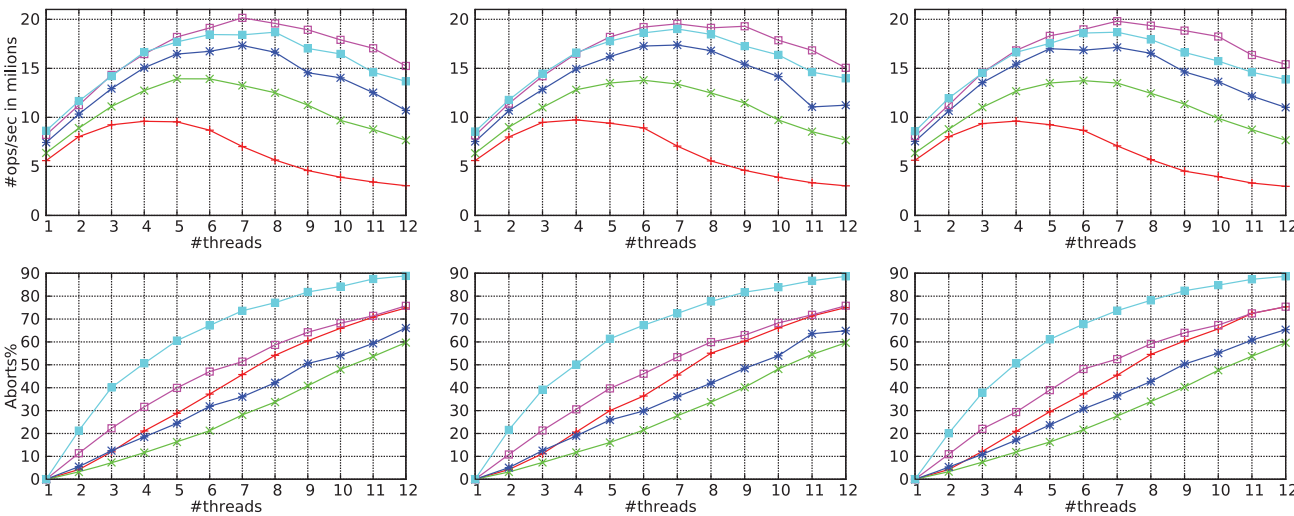

(g) $\mathrm{ur}=20 \%$; wlc $=0$

(h) $\mathrm{ur}=20 \%$; wlc $=100$

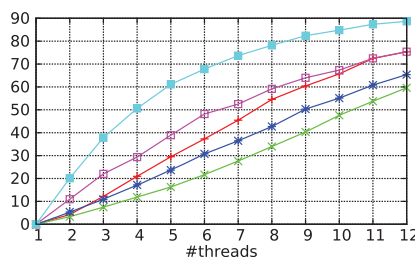

(i) $\mathrm{ur}=20 \%$; $w \mathrm{lc}=1,000$

Fig. 7. Hash table with $1 \%, 5 \%$, and $20 \%$ update rate (ur), and 0,100 , and 1.000 as work loop count (wlc). 


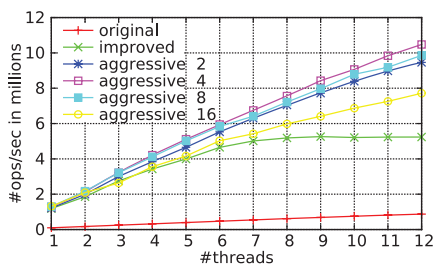

(a) $\mathrm{qpt}=1$

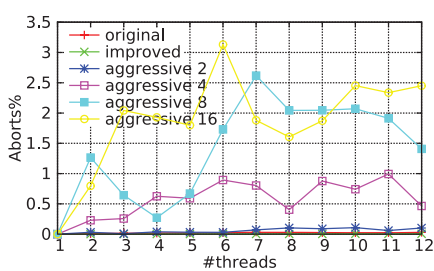

(d) $\mathrm{qpt}=1$

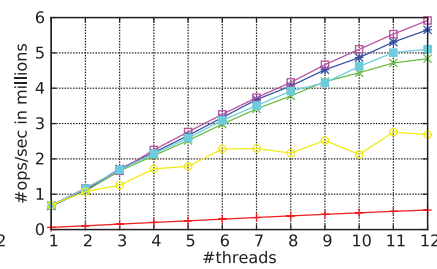

(b) $\mathrm{qpt}=2$

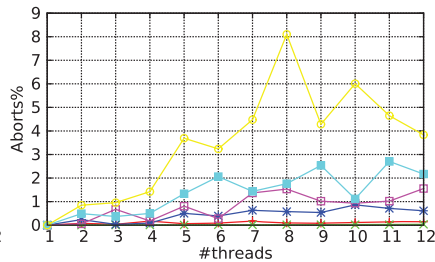

(e) $q p t=2$

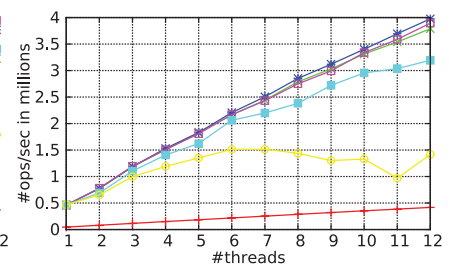

(c) $\mathrm{qpt}=3$

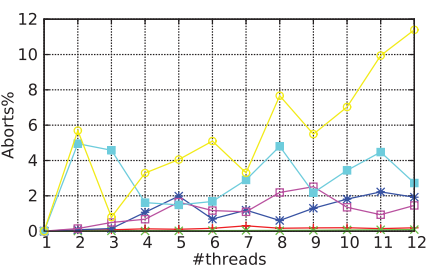

(f) $\mathrm{qpt}=3$

Fig. 8. Vacation: Throughput and abort rate for different numbers of queries per transaction (qpt).
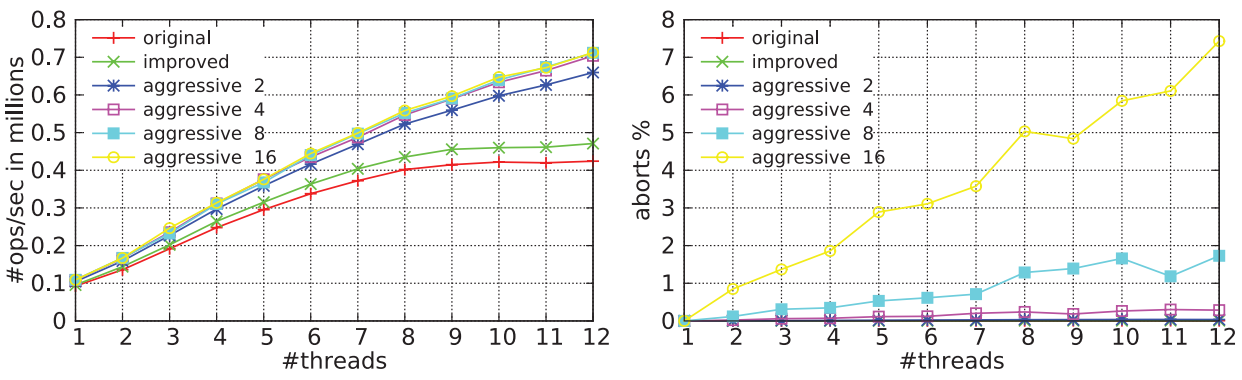

Fig. 9. SSCA2: Throughput (left) and abort rate (right)

Figure 8 shows graphs for different input values of queries per transaction (qpt). The size of transactions scale linearly with qpt. When qpt is low (Figure 8(a)), TC always improves the performance. In Figure 8(b), we show the performance for the default input value of qpt for the benchmark. When running with 12 threads, "aggressive 2" (the default aggressive factor) performs $19.4 \%$ better than "improved." The best case, "aggressive 4," performs $21.2 \%$ better than "improved," when running with 12 threads. However, when qpt is 3, "aggressive 8-16" perform worse than "improved" (Figure 8(c)) due to the high abort rate (Figure $8(f))$. The increased number of aborts negates the benefits of TC.

Figure 9 shows "original," "improved," and "aggressive" versions of SSCA2 benchmark. "Improved" is based on "original" where we modify the main loop, and instead of executing the transactions immediately, we buffer the values of the variables used in the transactions (explained in Section 5.1). These changes improved the performance of "original" slightly. We apply TC ("aggressive n") on the improved baseline ("improved") to show the benefit of using TC. The figure shows the performance of the parallel section of SSCA2 where "aggressive 2-16" have better scalability than "improved." When running with 12 threads, "aggressive 2" (the default aggressive factor) and "aggressive 16 " (the best case) perform better than "improved" by $36.4 \%$ and $47.4 \%$, respectively.

The default aggressive parameter ("aggressive 2") improves the performance of hash table, Vacation, and SSCA2 with negligible increase of the abort rate. The higher values 
of the aggressive parameter increase the abort rate considerably (Figures 7, 8, and 9) and can decrease performance ("aggressive 16" for Vacation, Figure 8). In the future, we plan to include the abort rate in the transaction coalescing heuristic to find the optimal aggressive factor.

\section{RELATED WORK}

Diniz and Rinard [1998] introduce lock coarsening (i.e., lock coalescing), a static analysis technique designed to automatically increase the lock granularity in object-based programs with lock protected methods. Lock coarsening achieves good performance by reducing the overhead introduced by mutex acquisition and mutex release. Lock coarsening is similar to TC because both techniques reduce the overheads of critical sections synchronization.

Chung et al. [2008] use STM to provide thread-safe dynamic binary translation. They translate all the code transactionally and create transactions at the basic block level. They reduce the overhead of transactional execution by merging consecutive basic blocks into a large transaction. However, the decision of merging is based on an instruction counter, rather than detailed analysis of transactions.

To address the overhead of STM, researchers have proposed hybrid TM systems that provide some hardware support for conflict detection in STM code [Saha et al. 2006; Minh et al. 2007; Shriraman et al. 2007]. For example, Saha et al. [2006] introduce Hardware Accelerated Software Transactional Memory (HASTM) as ISA extensions and novel hardware mechanisms that improve STM performance by using additional bits per cache line. In addition, minor hardware changes can improve performance of transactional applications significantly. Stipic et al. [2012] introduce TagTM, an STM implementation that reduces the overheads for metadata accesses.

Wang et al. [2012] describe a BlueGene/Q machine with best-effort HTM. BlueGene/Q maintains speculative states in the L2 cache and uses software register checkpointing with the setjmp () function. Even though BlueGene/Q has support for best effort hardware TM, this HTM implementation has issues with small transactions. The authors of the paper admit that the software register checkpointing has significant overhead for small transactions. We believe that TC can be complementary in reducing these overheads.

Many optimizations have been proposed for compilers and runtime systems to reduce the overheads of STM read and write operations [Wang et al. 2007; Wu et al. 2009; Dragojevic et al. 2009; Afek et al. 2011]. Wang et al. [2007] provide compiler optimizations for eliminating unnecessary read/write barriers (read after write barrier, barriers on local variables, etc.) and register checkpointing. Afek et al. [2011] propose static analysis and code motion to decrease the number of memory accesses. For eliminating redundant barriers and checkpointing, Wu et al. [2009] use compiler optimizations on statistics collected at runtime. Dragojevic et al. [2009] present runtime and compiler optimizations to identify transaction-local stack and heap allocation, and an API for annotating thread-local and read-only memory regions. All these techniques are complementary to TC because TC decreases the overheads associated with the initialization and committing of transactions.

Adl-Tabatabai et al. [2006] use compiler and runtime optimizations for transactional memory language constructs. They use a JIT compiler to reduce the overheads of STM. The JITting could be used also to find small and frequent transactions that benefit from transaction coalescing. Yu et al. [2010] provide an analytical model of the execution of transactional memory applications that analyzes the impact of TM design parameters including the conflict rate, number of checkpoints, and implementation overheads. This model could be used to analyze the benefits of transaction coalescing even before the program is executed. Zyulkyarov et al. [2012] introduce transaction profiling 
techniques to identify multiple potential conflicts from a single program run and the data structures involved in conflicts. Their techniques could be used to eliminate highly conflicting transactions from the candidates for coalescing.

Finally, He and Hong [2010] uses Continuous time Markov chains to describe the start and completion (commit or abort) of transactions. They analyze the mean transaction execution time to evaluate the performance of target transactional memory systems. Their use of Continuous time Markov chain is similar to the "transaction transition matrix" we use to find transaction pairs that benefit from transaction coalescing.

\section{CONCLUSIONS AND FUTURE WORK}

In this article, we showed that transaction coalescing can reduce transactional overheads. We introduced a profiling tool and a transaction coalescing heuristic for collecting and analyzing transactional information. We developed a profile-guided compiler pass that identifies and coalesces transactions, where the overheads of transactional start and commit are less than in the original transactions. We evaluated transaction coalescing using the STAMP applications and micro-benchmarks. For the default value of the aggressive factor, transaction coalescing improves the performance by $10.7 \%$ to $158.5 \%$ in hash table, by $19.4 \%$ in Vacation, and by $36.4 \%$ in SSCA2, when running with 12 threads. We showed that even better performance improvement can be achieved with larger aggressive factors. The improvement can go up to $21.2 \%$ in Vacation (for the aggressive factor equals 4) and up to $47.4 \%$ in SSCA2 (for the aggressive factor equals 16), when running with 12 threads.

As future work, we want to extend the transactional coalescing heuristic to find the optimal aggressive factor. One of the ideas is to include the abort rate because it might degrade the performance of benchmarks when transaction coalescing is too aggressive.

\section{REFERENCES}

Adl-Tabatabai, A.-R., Lewis, B. T., Menon, V., Murphy, B. R., Saha, B., and Shpeisman, T. 2006. Compiler and runtime support for efficient software transactional memory. In Proceedings of the 2006 ACM SIGPLAN Conference on Programming Language Design and Implementation (PLDI'06). 26-37.

AfeK, Y., Korland, G., AND Zilberstein, A. 2011. Lowering STM overhead with static analysis. In Proceedings of the Workshop on Languages and Compilers for Parallel Computing. 31-45.

BAder, D. AND MAdDURI, K. 2005. Design and implementation of the HPCS graph analysis benchmark on symmetric multiprocessors. In Proceedings of the 12th International Conference on High Performance Computing (HiPC'05). 465-476.

Chung, J., Dalton, M., Kannan, H., and Kozyrakis, C. 2008. Thread-safe dynamic binary translation using transactional memory. In Proceedings of the IEEE 14th International Symposium on High Performance Computer Architecture (HPCA'08). IEEE, 279-289.

Diniz, P. AND RINARD, M. 1997. Synchronization transformations for parallel computing. In Proceedings of the 24th ACM SIGPLAN-SIGACT Symposium on Principles of Programming Languages. ACM, 187-200.

DinIz, P. AND RINARD, M. 1998. Lock coarsening: Eliminating lock overhead in automatically parallelized object-based programs. Journal of Parallel and Distributed Computing 49, 2, $218-244$.

Dragojevic, A., Ni, Y., AND AdL-TABATABAI, A. 2009. Optimizing transactions for captured memory. In Proceedings of the 21st Annual Symposium on Parallelism in Algorithms and Architectures. ACM, 214-222.

Felber, P., Fetzer, C., ANd Riegel, T. 2008. Dynamic performance tuning of word-based software transactional memory. In Proceedings of the 13th ACM SIGPLAN Symposium on Principles and Practice of Parallel Programming. ACM, 237-246.

HARRIS, T., LARUS, J., AND RAJWAR, R. 2010. Transactional memory. Synthesis Lectures on Computer Architecture $5,1,1-263$.

He, Z. AND Hong, B. 2010. Modeling the run-time behavior of transactional memory. In Proceedings of the 2010 IEEE International Symposium on Modeling, Analysis Simulation of Computer and Telecommunication Systems. 307-315. 
Herlihy, M. AND Moss, J. E. B. 1993. Transactional memory: Architectural support for lock-free data structures. In Proceedings of the 20th Annual International Symposium on Computer Architecture (ISCA'93).289-300.

GCC. 2013. GCC, the GNU Compiler Collection. Retrieved from http://gcc.gnu.org/.

Minh, C., Chung, J., Kozyrakis, C., And Olukotun, K. 2008. STAMP: Stanford transactional applications for multi-processing. In Proceedings of the IEEE International Symposium on Workload Characterization (IISWC'08). IEEE, 35-46.

Minh, C. C., Trautmann, M., Chung, J., McDonald, A., Bronson, N., Casper, J., Kozyrakis, C., and Olukotun, K. 2007. An effective hybrid transactional memory system with strong isolation guarantees. In Proceedings of the 34th Annual International Symposium on Computer Architecture (ISCA'07).69-80.

Necula, G., McPeak, S., Rahul, S., AND Weimer, W. 2002. CIL: Intermediate language and tools for analysis and transformation of C programs. In Compiler Construction. Springer, 209-265.

Saha, B., Adl-Tabatabai, A., And Jacobson, Q. 2006. Architectural support for software transactional memory. In Proceedings of the 39th Annual IEEE/ACM International Symposium on Microarchitecture (MICRO'06). IEEE, 185-196.

Shriraman, A., Spear, M. F., Hossain, H., Marathe, V. J., Dwarkadas, S., and Scott, M. L. 2007. An integrated hardware-software approach to flexible transactional memory. In Proceedings of the 34th Annual International Symposium on Computer Architecture (ISCA'07). 104-115.

Stipic, S., Tomic, S., Zyulkyarov, F., Cristal, A., Unsal, O., And Valero, M. 2012. TagTM-Accelerating STMs with hardware tags for fast meta-data access. In Proceedings of the Design, Automation Test in Europe Conference Exhibition (DATE'12). 39-44.

Wang, A., Gaudet, M., Wu, P., Amaral, J. N., Ohmacht, M., Barton, C., Silvera, R., and Michael, M. 2012. Evaluation of blue gene/Q hardware support for transactional memories. In Proceedings of the 21st International Conference on Parallel Architectures and Compilation Techniques. ACM, 127-136.

Wang, C., Chen, W., Wu, Y., Saha, B., and Adl-Tabatabai, A. 2007. Code generation and optimization for transactional memory constructs in an unmanaged language. In Proceedings of the 2007 International Symposium on Code Generation and Optimization (CGO'07). IEEE, 34-48.

Wu, P., Michael, M., von Praun, C., Nakaike, T., Bordawekar, R., Cain, H., Cascaval, C., Chatterjee, S., Chiras, S., Hou, R., ET AL. 2009. Compiler and runtime techniques for software transactional memory optimization. Concurrency and Computation: Practice and Experience 21, 1, 7-23.

Yu, X., He, Z., AND Hong, B. 2010. An analytical model on the execution of transactional memory. In Proceedings of the 22nd International Symposium on Computer Architecture and High Performance Computing (SBAC-PAD'10). IEEE, 175-182.

Zyulkyarov, F., Stipic, S., Harris, T., Unsal, O., Cristal, A., Hur, I., and Valero, M. 2010. Discovering and understanding performance bottlenecks in transactional applications. In Proceedings of the 19th International Conference on Parallel Architectures and Compilation Techniques. ACM, 285-294.

Zyulkyarov, F., Stipic, S., Harris, T., Unsal, O., Cristal, A., Hur, I., and Valero, M. 2012. Profiling and optimizing transactional memory applications. International Journal of Parallel Programming 40, 1 , 25-56.

Received November 2013; revised November 2013; accepted November 2013 